Address for Correspondence: Dr. Guangsheng He, MD, The First Affiliated Hospital of Nanjing Medical University, Jiangsu Province Hospital Nanjing 210029, Jiangsu Province, China.

E-mail: heguangsheng@medmail.com.cn

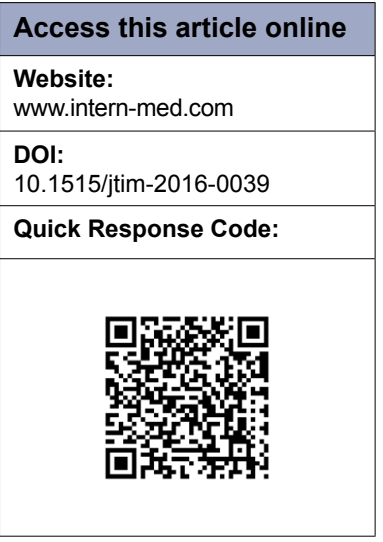

\title{
The third-time chronic myeloid leukemia in lymphoblastic crisis with ABL1 kinase mutation induced by decitabine, dexamethason combined with nilotinib and dasatinib
}

\author{
Suli Wang, Chun Qiao, Yu Zhu, Wenyi Shen, Guangsheng He, Jianyong Li \\ The First Affiliated Hospital of Nanjing Medical University, Jiangsu Province Hospital, \\ Nanjing 210029, Jiangsu Province, China
}

\section{ABSTRACT}

Blast crisis $(\mathrm{BC})$ is the major remaining challenge in the management of chronic myeloid leukemia $(\mathrm{CML})$. The prognosis of the $B C$ patient who carries $A B L$ kinase mutation is very poor. One patient, with lymphoid CML-BC third time, was detected with T315A/F359I/M244V compound mutation by direct sequencing after treatment with tyrosine kinase inhibitions three years. The patient was treated with decitabine, dexamethasone, in combination with nilotinib and dasatinib. Then this patient received a complete hematologic response and cytogenetic response after two cycles of treatment.

Key words: chronic myeloid leukemia, T315A, F359I, M244VA, lymphoid blast crisis, decitabine

In June 2009, a 45-year-old female was hospitalized after two weeks of dizziness and weakness. Blood test showed white blood cell (WBC) count of $200.0 \times 10^{9} / \mathrm{L}$, and the hemoglobin $(\mathrm{Hb})$ level and platelet count were in normal scope. Chronic myeloid leukemia - crisis phase (CML$\mathrm{CP}$ ) was diagnosed on bone marrow examination, Ph chromosome and the BCRABL fusion gene. She was given $400 \mathrm{mg}$ Imatinib Mesylate (IM) daily, and she achieved complete hematologic response (CHR) and cytogenetic response (CCyR) after three months. In June 2012, there was a significant increase in the number of the WBC of this patient. The bone marrow smear revealed lymphoid blast crisis of CML, she was treated with nilotinib $400 \mathrm{mg}$ twice daily for three months and obtained CHR. In July 2013, she was diagnosed with CML-BC for the second time, although with the maintenance therapy of nilotinib. F359I mutation was detected in ABL kinase domain, the treatment was changed to dasatinib $140 \mathrm{mg}$ daily. After five months, she achieved CHR once again.
On 24 ${ }^{\text {th }}$ March 2014, the patient came to our hospital with fever and weakness, which she had been suffering from since past one week. The blood examination showed $\mathrm{Hb}$ level of $78 \mathrm{~g} / \mathrm{L}$, WBC count of $3.41 \times 10^{9} / \mathrm{L}$ and platelet count of 66 $\times 10^{9} / \mathrm{L}$. The bone marrow smear revealed $90.4 \%$ primitive and immature lymphocytes. And the flow cytometry (FCM) analysis showed that $74.5 \%$ of the nucleated cells were malignant lymphoid blasts, expressing human leukocyte antigen-DR (HLA-DR), CD13, CD38, CD19, CD33, CD10, CD34. The BCR-ABL (P210) fusion gene had a quantitative result of $149.0 \%$. The BCRABL fusion gene kinase domain mutation analysis showed T315A, F359I, M244V mutations. Lymphoid blast crisis of CML was definitely diagnosed.

The patient suffered high fever with a body temperature of $39^{\circ} \mathrm{C}$ and with a saturation of pulse oxygen less than 95\%. Chest CT showed severe pneumonia. Considering the patient's intolerance of combined chemotherapy, and the mutation T315A, 
which is resistant to dasatinib and F359I that is less sensitive to nilotinib, ${ }^{[1]}$ we tried to treat the patient with decitabine (DAC) and dexamethasone, combining it with nilotinib and dasatinib (DAC $20 \mathrm{mg} / \mathrm{m}^{2}$ over $1 \mathrm{~h}$ daily on day 1 to 5 , dexamethason $10 \mathrm{mg}$ daily on day 1 to 10 , nilotinib 400 $\mathrm{mg}$ and dasatinib $70 \mathrm{mg}$ orally daily). Then the patient was administered CHR with bone marrow smear, which detected no primitive and immature lymphocytes. The blood test identified $\mathrm{Hb}$ level of $70 \mathrm{~g} / \mathrm{L}$, WBC count of $4.70 \times 10^{9} / \mathrm{L}$ and platelet count of $37 \times 10^{9} / \mathrm{L}$.

On 10th April 2014, the patient was given a consolidation treatment. As a result, only $2.0 \%$ immature cells were discovered in the bone marrow smear. Minimal residual disease (MRD) was 2.3 × 10-4 (malignant clone expressed CD45dim, CD34+, CD19+, CD10+, CD20-/dim, CD13, $\mathrm{CD} 3+/ \mathrm{dim})$ by flow cytometry. Although the T315 A/ F359I/M244V mutations were still positive, the quantity of BCR-ABL (P210) fusion gene was $8.98 \%$, and the bone marrow cell chromosome was 46, XX [20].

\section{DISCUSSION}

Under the tyrosine kinase inhibitions (TKIs) therapy, there are still a number of patients with CML who progress to BC. How this leukemia transforms from a relatively indolent $\mathrm{CP}$ to an aggressive $\mathrm{BC}$ is still unclear. ${ }^{[2]}$ Once $\mathrm{BC}$ happens, the management depends on the type of leukemia (myeloid or lymphoid) and if they successfully achieve CR or not. ${ }^{[3]}$ Allogeneic stem cell transplantation (AlloHSCT) may be the only curly therapy for the patients, ${ }^{[4]}$ but as influenced by a donor source and recipient status, it is successful in only a minority of CML-BC patients. ${ }^{[5]}$

This patient was diagnosed with CP and was given IM 400 . Three years later, she progressed to $\mathrm{BC}$ with compound BCR-ABL1 kinase domain mutations of T315A, F359I, $M 244 \mathrm{~V}$ by direct sequencing. The mutation T315A is resistant to dasatinib, F359I is less sensitive to nilotinib, and $M 244 V$ is sensitive both to dasatinib and nilotinib. ${ }^{[1]}$ There was no standard therapy for these patients with BCR-ABL1 kinase domain mutations. Since this patient still progressed to $\mathrm{BC}$ during being treated by dasatinib and nilotinib orderly, it was suggested that the mechanisms of resistance or BC were more than ABL1 kinase domain mutation. So, we had to seek the novel therapy for her.

In CML, DNA hypermethylation was in concert with disease progression. ${ }^{[6]} \mathrm{DAC}$, as one of hypomethylating agents, is a cytosine analog causing DNA methyltransferase (DNMT) inhibition. Beside its single-agent efficiency in CML, including imatinib-resistant cases, it has been studied in various combination treatment regimens. ${ }^{[7]}$
Schenekburger et al found DAC could trigger differentiation, senescence, and autophagy in CML K-562amd MEG-01 cell lines at clinically relevant concentration, respectively. ${ }^{[8]}$ Prolonged treatment of DAC could induce mitochondrialdependent apoptosis in CML cells. The combinatory treatment of relatively resistant CML with DAC and either cisplatin or etoposide increased apoptosis synergistically. ${ }^{[8]}$

DAC was also able to decrease telomere length, to reduce telomerase activity and to decrease human telomerase reverse transcriptase (hTERT) expression through decreased binding of c-myc to the hTERT promoter. ${ }^{\left[{ }^{[9]}\right.}$ Then, it induces senescence in CML cell lines.

In Kantarjian's report, ${ }^{[10]} 130$ patients with CML were treated with high dose of DAC. DAC was given at 100 $\mathrm{mg} / \mathrm{m}^{2}$ over 6 hours every 12 hours for 5 days to the first 13 patients, $75 \mathrm{mg} / \mathrm{m}^{2}$ to the subsequent 33 patients, and $50 \mathrm{mg} / \mathrm{m}^{2}$ to the remaining 84 patients. Of the 64 patients in BC, 6 patients $(9 \%)$ achieved CHR and 5 patients $(8 \%)$ achieved CCyR. The high dose of DAC appeared to have significant anti-CML activity.

Since this patient had experienced her third BC and her case was complicated due to her suffering from pneumonia, we did not choose a high dose DAC to avoid serious myelosuppression. As nilotinib and dasatinib can be targeted at T315 A and F359I respectively, and the mutation of $M 244 \mathrm{~V}$ is sensitive to both TKIs, we combined these two TKIs with DAC and dexamethason. The patient achieved CHR and CCR after two cycles of therapy.

We infer that DAC combined with correspondingly sensitive TKIs for these patients who progress into CML$\mathrm{BC}$ with $\mathrm{ABL}$ kinase mutations may have clinical activity. This warrants further clinical exploration.

\section{Conflict of Interest}

None declared.

\section{REFERENCES}

1. Kim SH. Kim D, Kim DW, Goh HG, Jang SE, Lee J, et al. Analysis of Bcr-Abl kinase domain mutations in Korean chronic myeloid leukemia patients: poor clinical outcome of P-100p and T315I mutation is disease phase dependent. Hematol Oncol 2009;27:190-7.

2. Chereda B, Mole JV. Natural course and biology of CML. Melo Ann Hematol 2015;94(S2): S107-21.

3. Saueles S, Silver RT. Management of chronic myeloid leukemia in blast crisis. Ann Hematol 2015; 94(S2): S159-65.

4. Hehlmann R. How I treat CML blast crisis. Blood 2012;120:737-47.

5. Baccarani M, Saglio G, Goldman J, Hochhaus A, Simonsson B, Appelbaum $\mathrm{F}$, et al. Evolving concepts in the management of chronic myeloid leukemia: recommendations from an expert panel on behalf of the European Leukemia Net. Blood 2006; 108: 1809-20. 
6. Nguyen TT, Mohrbacher AF, Tsai YC, Groffen J, Heisterkamp N, Nichols $\mathrm{PW}$, et al. Quantitative measure of c-abl and $\mathrm{p} 15$ methylation in chronic myelogenous leukemia: biological implications. Blood 2000;95:2990-2.

7. Issa JP, KantarJian HP. Introduction emerging role of epigenetic therapy: Focus on Decitabine. Semin Hematol 2005;42(3 Suppl 2):S1-2.

8. Schnekenburger M, Grandjenette C, Ghelfi G, Karius T, Foliguet B, Dicato $\mathrm{M}$, et al. Sustained exposure to the DNA demethylating agent, 2'-deoxy-5-azacytidine, leads to apoptotic cell death in chronic myeloid leukemia by promoting differentiation, senescence, and autophagy. Biochem Pharmacol 2011; 81: 364-78.

9. Grandjenette C, Schnekenburger M, Karius T, Ghelfi J, Gaigneaux A, Henry E, et al. 5-aza-2-deoxycytidine- mediated c-myc Down-regulation
Triggers Telomere-dependent Senescence by Regulating Human Telomerase Reverse Transcriptase in Chronic Myeloid Leukemia. Neoplasia 2014;16:511-28.

10. Kantarjian HM, O’Brien S, Cortes J, Giles FJ, Faderl S, Issa JP, et al. Results of decitabine (5-aza-2'deoxycytidine) therapy in 130 patients with chronic myelogenous leukemia. Cancer 2003; 98:522-8.

How to cite this article: Wang S, Qiao C, Zhu Y, Shen W, He G, Li J. The third-time chronic myeloid leukemia in lymphoblastic crisis with ABL1 kinase mutation induced by decitabine, dexamethason combined with nilotinib and dasatinib. J Transl Intern Med 2016; 4: 182-84. 